\title{
The immediate and long-term effect on runway performance of reversing ethanol and dextrose conditions
}

ROBERT L. SCHALOCK ${ }^{1}$ AND KEITH A. WOLLEN

WASHINGTON STATE UNIVERSITY

Shifting from alcohol to dextrose or dextrose to alcohol conditions produced the following results: At the reversal point, shifts in either condition resulted in no performance decrement. After the reversal, running speed increased if shifted from alcohol to dextrose, and decreased if shifted from dextrose to alcohol.

Studies dealing with the effects of shifting from placebo to alcohol or from alcohol to placebo conditions have resulted in apparent discrepancies. On the one hand, Denenberg, Pawlowski, \& Zarrow (1961) report that shifting from placebo to alcohol did not result in a response decrement; whereas Barry \& Miller (1962), Miller \& Miles (1936), and Nelson \& Wollen (1965) report a response decrement after the shift to alcohol. On the other hand, Nelson \& Wollen (1965) also report that shifting from alcohol to placebo results in a greater response decrement than shifting from placebo to alcohol.

These contradictory findings and their explanations are difficult to interpret due to: Lack of non-reversed control groups (Nelson \& Wollen, 1965); inadequate control of injected alcohol (Denenberg et al, 1961); and use of alcohol solutions whose concentrations not only differ, but were potentially too weak to reflect the alcohol's effects. The study reported below is a further attempt to determine the effects of shifting from alcohol to placebo, and vice versa, by controlling the weaknesses reported above.

\section{Subjects}

\section{METHOD}

The Ss were 40 naive, male, hooded rats from the Washington State University colony. The Ss, which ranged from 90-116 days old at the beginning of deprivation, were maintained on a $23-\mathrm{hr}$. food and water deprivation schedule.

\section{Apparatus}

The apparatus used was a straight alley $60 \mathrm{in.}$ long, divided into a 12-in. start box (SB), a 36-in. alley, and a 12-in. goal box (GB). Inside alley dimensions were 3.5 in. wide and $4.7 \mathrm{in}$. high. The apparatus was painted flat black throughout, and the entire runway was covered by elear Plexiglas. Two guillotine doors separated the SB from the alley. The door nearest the SB was transparent, whereas the one nearest the alley was opaque. An opaque door separated the GB from the runway.

\section{Pretraining}

Pretraining consisted of (1) handling each animal $3 \mathrm{~min}$. daily from the fifth to the fourteenth day of deprivation; (2) feeding each animal $1097 \mathrm{mg}$ Noyes reward pellets immediately prior to his daily ad lib feeding from the tenth to the fourteenth day of deprivation; (3) injecting each animal with ethanol (1.5 cc of a $30 \%$ by volume ethanol and distilled water solution per $100 \mathrm{gm}$ of body weight) from Day 10 to Day 14 of deprivation; and (4) allowing Ss, in groups of 3 , to run freely in the apparatus for an hour per day from Days 11 to 13 of deprivation, and alone for $15 \mathrm{~min}$. on Day 14.

\section{Training}

During training each $\mathrm{S}$ was weighed immediately before running, and depending upon the group to which he belonged was injected with $1.5 \mathrm{cc} / 100 \mathrm{gm}$ of either a $30 \%$ ethanol and water solution or a caloricequivalent $(54.86 \mathrm{gm} / 100 \mathrm{ml}$ of water) dextrose solution. Both solutions were heated to $101^{\circ}$ before being given. Fluid administered was by stomach loading, using a Baxter K-31 Infant Feeding Tube with syringe.

The training phase consisted of 45 trials per $S, 5$ the first day and 10 on each of the 4 successive days. The procedure for each trial was as follows. The $S$ was taken from a waiting cage and placed in the start box. The opaque door was opened as soon as the $\mathrm{S}$ oriented towards it. Exactly $1 \mathrm{sec}$. later, the second (transparent) door was raised automatically. When the $S$ reached the GB, the GB door was lowered to prevent retracing. Following the consumption of one $97 \mathrm{mg}$ Noyes pellet the animal was placed in a waiting cage for an intertrial interval of $70 \mathrm{sec}$, following which another trial was given.

The response measure used was the time required to run from the interception of a light-beam 6 in. from the SB until the $S$ intercepted a second beam, 24 in. farther down the alley. Following each day's 10 trials, Ss were given the opposite solution from that given before they were mun.

\section{Post-shift}

During the post-shift phase, which included 10 trials per day for 5 days, half of the Ss in each group (alcohol and dextrose) were changed to the opposite condition, whereas the other half of each group continued to receive the same solution. Each group was designated by two letters indicating the pre- and post-shift condition re- 
spectively, i.e., AA, AD, DA, and DD. The study was run in 5 replications, with each replication consisting of 2 Ss in each of the 4 groups. Within each replication, Ss were assigned so that the mean weights for the 4 groups would be approximately equal.

\section{RESULTS AND DISCUSSION}

Figure 1 presents the running speed (ft. $/ \mathrm{sec}_{\text {. }}$ ) data for Days 1 and 5 which were analyzed by means of a design in which experimental conditions were a between factor and days a within factor. Since the analysis revealed a significant days by drugs interaction $(F=11.46$, $\mathrm{df}=1 / 38, \mathrm{p}<.005$ ) analyses were conducted on individual days. There was a significant difference between the two groups on Day $1(F=23.38, d f=1 / 38, p<.001)$, but not on Day $5(F<1)$. This initial superiority of the alcohol group has been found by other investigators (Barry, Wagner, \& Miller, 1962, and Denenberg et al, 1961) but was not expected in the present study since pretraining was instituted to reduce the initial fear of the apparatus. In spite of the pretraining, the results suggest that the adaptation was insufficient to reduce the Ss' fear and, consequently, the initial superior performance of the alcohol Ss was probably due to the fear-reducing properties of alcohol (Barry et al, 1962).

Analysis of the data from Days 5 and 6 indicate

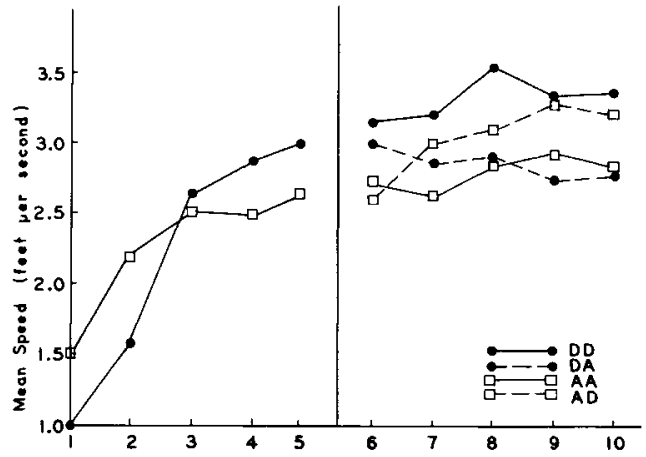

Fig. 1. Mean running speeds as a function of experimental conditions. that shifting from alcohol to dextrose or vice versa produced no change in running speed $(F<1)$. These data suggest that with adaptation to the apparatus and alcohol, there is no immediate effect of reversing the experimental conditions.

If one analyzes the post-reversal data for Groups $\mathrm{DA}$ and $\mathrm{AD}$, then an effect of reversing conditions is apparent. Analysis of the running speed data for Days 6 and 10 indicated a significant days by drugs interaction $(\mathrm{F}=4.17, \mathrm{df}=3 / 36, \mathrm{p}<.025)$. In addition, Group $A D$ 's running speed increased significantly from Day 6 to Day $10(\mathrm{~F}=13.65, \mathrm{df}=1 / 9, \mathrm{p}<.005)$, but the decline in Group DA's speed did not reach significance $(F=3.25, \mathrm{~d} f=1 / 9, \mathrm{p}>.10)$. Therefore, it appears as though the long-term effect of reversing conditions is an increase in running speed if shifted from alcohol to dextrose, but no effect if shifted from dextrose to alcohol. However, the significant difference between Groups DA and DD on Days 9 and $10(t=2.18, \mathrm{df}=1 / 9$, $p<.05)$ suggests that shifts from dextrose to alcohol eventually result in a performance decrement.

\section{References}

Barry, H. III, \& Miller, N. E. Effects of drugs on approach-avoidance conflict tested repeatedly by means of a "telescope alley." J. comp. physiol. Psychol., 1962, 55, 201-210.

Barry, H. III, Wagner, A. R., \& Miller, N. E. Effects of alcohol and amabartital on performance inhibited by experimental extinction. J. comp. physiol. Psychol., 1962, 55, 464-468.

Denenberg, V. H., Pawlowski, A. A., \& Zarrow, M. X. Prolonged alcohol consumption in the rat. I. Acquisition and extinction of a bar pressing response. Quart. J. Stud. Alc., 1961, 22, 14-21.

Miller, N. E., \& Miles, W. R. Alcohol and removal of reward: An analytical study of rodent maze behavior. J. comp. Psychol., $1936,21,179$.

Nelson, P. B., \& Wollen, K. A. Effects of ethanol and partial reinforcement upon runway acquisition. Psychon. Sci., 1965, 3, 135136.

\section{Note}

1. Now at the Psychophysiology Lab., VA Hospital, American Lake, Washington 98493 . 